CDD: 029.7

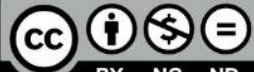

\title{
CONSPECTUS: \\ UM MÉTODO PARA O GERENCIAMENTO DE COLEÇÕES EM BIBLIOTECAS
}

CONSPECTUS:

A TOOL FOR COLLECTION MANAGEMENT IN LIBRARIES

\section{Rita de Cássia do Vale Caribé ${ }^{1}$}

Resumo: Apresenta a evolução histórica, conceitos e descrição da metodologia Conspectus, desenvolvida nos Estados Unidos na década de 1980. Essa metodologia é utilizada para o desenvolvimento de coleções, para a avaliação qualitativa de coleções e como instrumento para o gerenciamento de coleções em diversas bibliotecas no mundo.

Palavras-chave: Conspectus. Avaliação de coleção. Desenvolvimento de coleção.

Gerenciamento de coleção.

Abstract: Presents the historical evolution, concepts and description of the Conspectus methodology, developed in the United States in the 80s. This methodology is applied in collections development, qualitative collection's evaluation, as well as for collection management in many libraries worldwide.

Keywords: Methodology. Collection evaluation. Collection managemente. Collection development.

\footnotetext{
${ }^{1}$ Professora adjunta da Faculdade de Ciência da Informação da Universidade de Brasília (UnB). Bibliotecária graduada em 1979 pela UFMG, Mestre em Biblioteconomia e Documentação pela UnB em 1989, doutora em Ciência da Informação pela UnB em 2011. Analista administrativa do Ibama aposentada em 2011. Brasil. E-mail: rita.caribe@ gmail.com Recebido em: 19/05/2013 - Aceito em: 18/09/2013.
} 


\section{INTRODUÇÃO}

Este estudo tem como objetivo apresentar e descrever a metodologia Conspectus que vem sendo utilizada como instrumento para auxiliar o gerenciamento de coleções, desde a década de 1980, em diversos países do mundo - Estados Unidos, Canadá, Inglaterra, Nova Zelândia, Grécia, Checoslováquia e Peru. Essa metodologia está descrita, de forma sucinta, no documento Guidelines for collection development policy using the Conspectus model, publicado pela International Federation of Library Associations and Institutions (IFLA); consta também de documentos da American Library Association (ALA) e da Online Computer Library Center (OCLC).

Em pesquisa realizada na base de dados LISA com o termo conspectus foram recuperadas apenas 230 referências, e nem todos os artigos tratavam do conspectus que está sendo apresentado neste artigo. Na literatura brasileira, de forma surpreendente, não foi identificada, nenhuma citação ou comentário sobre a mesma, nem relatos sobre a sua utilização.

Assim, este estudo vem preencher essa lacuna na literatura do país, apresentando a metodologia com a finalidade de provocar discussões quanto à sua utilização também em bibliotecas brasileiras. Os aspectos relacionados a críticas e experiências de implementação, estudos de caso dessa metodologia em outras bibliotecas estrangeiras não são tratados neste estudo.

\section{GERENCIAMENTO DE COLEÇÕES}

As bibliotecas existem há aproximadamente 5.000 anos, desde então sempre houve um processo, formal ou informal, composto por critérios, estratégias e metas, para coletar os objetos de informação que deveriam ser incorporados às coleções dessas unidades. Na literatura estrangeira observa-se que na década de 1920, esse era denominado processo de seleção; em um segundo momento, por volta das décadas de 1960 e 1970, de desenvolvimento de coleções (collection development) e, a partir da década de 1980, de gerenciamento de coleções (collection management). De maneira geral, este fato constitui um processo universal, desenvolvido em qualquer tipo de unidade de informação - biblioteca, centro de informação ou centro de documentação - bem como por qualquer instituição que esteja interessada em desenvolver algum 
tipo de coleção, de base de dados ou em fornecer um serviço ou produto de informação. Assim, qualquer instituição deverá, necessariamente, adotar um conjunto de critérios, estratégias e metas para coleta, seleção e descarte dos objetos de informação que pretende incorporar à sua coleção, de forma dinâmica e constante, bem como deverá estabelecer formas, metodologias e instrumentos para avaliar essas coleções (EVANS; SAPONARO, 2005).

$\mathrm{Na}$ literatura estrangeira há uma quantidade enorme de publicações, incluindo pelo menos quatro periódicos especializados no tema desenvolvimento e gerenciamento de coleções (Library Acquisitions: practices and theory; Library Collections, acquisitons \& technical services; Collection Building; The Acquisitions Librarian), há também divisões na IFLA - Comitê Permanente da Seção de Aquisições e Desenvolvimento de Coleções - e na ALA cuja missão está voltada para esse tema, além de livros densos, pesquisas. No Brasil, lamentavelmente, pesquisa-se e escreve-se pouco sobre o assunto.

\section{CONSPECTUS: ASPECTOS HISTÓRICOS}

Devido à explosão informacional que ocorreu nos Estados Unidos, acompanhada da redução de recursos financeiros alocados às bibliotecas a partir da década de 1970, à redução dos espaços físicos destinados às bibliotecas, houve a necessidade, principalmente por parte das bibliotecas universitárias, de desenvolver instrumentos que facilitassem a cooperação entre elas. Foi necessário sistematizar as diferentes experiências vivenciadas pelas bibliotecas que já haviam identificado ou caracterizado suas coleções. Ao mesmo tempo, os bibliotecários se viram envolvidos com tarefas de gerenciamento de coleções (BUSHING, 2001; MUNROE; STEEG, 2004).

Johnson (2004) afirma que, na década de 1970, os estudos qualitativos de coleção, não possuíam uma terminologia padronizada. Em 1979, foi publicado pela ALA o Guidelines for Collection Development, que consistiu no primeiro esforço de desenvolver um vocabulário compartilhado para descrever intensidade-força (strength) da coleção utilizando níveis. Esse trabalho definiu cinco níveis, os quais podem ser aplicados à coleção existente - densidade da coleção (collection density) e à atividade de coleta da coleção - intensidade (collection intensity). Esses níveis 
eram: A - Nível abrangente (Comprehensive Level); B - Nível de pesquisa (Research Level); C - Nível de estudo (Study Level), D - Nível básico (Basic Level) e E - Nível mínimo (Minimal Level).

Nesse ambiente, um grupo de bibliotecas que formavam o Research Library Group (RLG), aperfeiçoou o trabalho da ALA, invertendo a ordem dos níveis e acrescentando mais um nível - fora do escopo (out-of-scope). O grupo foi responsável pelo desenvolvimento do conceito e da infra-estrutura no começo dos anos 1980, que consistia, inicialmente, de um inventário em que era registrada a coleção existente especificando suas forças e intensidade, utilizando planilhas baseadas no esquema de classificação da Library of Congress $(L C)$. Assim, foi criada a metodologia Conspectus para ser utilizada, principalmente em grandes bibliotecas.

Em 1982, como resultado do trabalho desenvolvido por bibliotecas universitárias, nas áreas de avaliação de coleções, o RLG Conspectus Online foi introduzido, bem como foram incorporadas as experiências desses profissionais na área de coleta (BRIEF, 1997). Nesse mesmo ano, a grande maioria das bibliotecas universitárias dos Estados Unidos já o utilizava, pois compreenderam que a ferramenta lhes permitia definir sua coleção, ou uma parte dela, e compará-la com outras coleções, independentemente de seu tamanho ou de suas características bibliográficas (JOHNSON, 2004; MUNROE; STEEG, 2004). Nesta mesma época, foi adotado pela Association of Research Libraries (ARL), pelo North American Collections Inventory Project. Foi adaptado pela National Library of Canadá para seu uso naquele país, e inserido no Reino Unido, bem como em outros países da Europa e Austrália. O RLG Conspectus tornou-se uma ferramenta de avaliação de coleções largamente reconhecida.

Inicialmente, o Conspectus foi desenvolvido como um instrumento para auxiliar a construção de coleções de maneira cooperativa. Porém, a metodologia expandiu para servir, também: como instrumento para o planejamento do empréstimo entre bibliotecas; como um documento público para o uso pela instituição; como instrumento de relacionamento com o público e para negociação de orçamentos; como uma estrutura sobre a qual desenvolve-se a política de desenvolvimento de coleções; como estímulo para buscar financiamentos; como instrumento de treinamento; como uma fonte para creditar a informação; como um meio para dar segurança ao 
bibliotecário, e como base para distribuição e preservação de responsabilidades (BUSHING, 2001; TALAVERA IBARRA, 2005).

Originalmente, a ferramenta consistia de uma lista de diferentes temas, separados em divisões, categorias e descritores juntamente com a classificação da Library of Congress (LC), aos quais se agregavam os níveis de intensidade e os idiomas conforme a metodologia. Cabe ressaltar que a LC também utiliza a metodologia Conspectus.

Em seguida, o grupo de bibliotecas que integrava o Pacific Norwest Project apresentou propostas de modificações com o objetivo de adaptar o Conspectus para uso em bibliotecas de menor porte, em bibliotecas públicas por exemplo. Para isso adaptaram a ferramenta para sua utilização com a Classificação Decimal de Dewey (CDD), criaram uma base de dados com todas as categorias de assunto, possibilitando às bibliotecas gerir automaticamente seus dados e coleção. Outra inovação incluída por esse grupo foi a possibilidade de ampliar os códigos de nível com subdivisões (2a, 2b, 3a, 3b) que são detalhadas a seguir. Posteriormente, os integrantes do grupo Pacific Norwest Project formaram o grupo Western Library Network (WLN) que passou a ser o responsável pelo Conspectus. A WLN incluiu mais outras inovações e adaptações para atender às necessidades de diferentes tipos de bibliotecas, incluindo as 24 divisões, as 500 categorias e aproximadamente quatro mil descritores.

Em 1999, a WLN se uniu ao Online Computer Library Center (OCLC) e passou a ser uma ferramenta totalmente automatizada. O manual foi publicado naquele mesmo ano, sob o título Using the Conspectus method: a collection assessment handbook; nele foram criadas novas categorias de assunto e outros conspectus para atender a necessidades específicas como a área médica e música.

\section{CONSPECTUS: CONCEITOS}

O termo conspectus, em inglês, significa um levantamento geral por assunto, uma sinopse, uma revisão, um resumo, um levantamento (survey) ou fazer uma revisão geral. O termo, no entanto, é oriundo do latim, especificamente o particípio passado de conspicere (conspic+ere), que significa observar, perceber, avisar. Quaisquer dessas acepções podem ser aplicadas às atividades a serem realizadas com a ajuda dessa ferramenta. 
De acordo com a Biblarz, et al. (2001) o Conspectus significa uma visão geral ou um resumo da profundidade da coleção e sua organização por assunto de acordo com um sistema de classificação ou pela combinação de ambos. É uma sinopse da coleção ou uma visão geral da política coordenada do desenvolvimento de coleções de um consórcio, de uma rede ou de uma biblioteca individualmente. O Conspectus inclui, também, os códigos padronizados para os níveis da coleção e os idiomas dos materiais que o integram.

Para Munroe e Steeg (2004) o Conspectus é um processo organizado de análise sistemática e de descrição da coleção que utiliza padrões definidos. É também considerado um conjunto de códigos, um instrumento, um meio para se chegar a um determinado fim, um levantamento, uma visão geral ou um esboço a ser utilizado para avaliações sistemáticas. Esses dois autores afirmam que há um consenso entre diversos especialistas de que o Conspectus é uma metodologia ou um instrumento que tem por objetivo ser sistemático e prover definições de padrões. Johnson (2004) corrobora essa afirmativa quando comenta a necessidade de padrões para tratar das avaliações qualitativas da coleção.

De acordo com a IFLA, uma política de desenvolvimento de coleções deve, necessariamente, possuir um item que descreva a coleção, indicando seus pontos fortes e fracos. É nesse ponto que se insere o Conspectus, como um instrumento de apoio à elaboração de políticas de desenvolvimento de coleções que estejam estruturadas por áreas temáticas para indicar a intensidade ou a profundidade da coleção; como instrumento indicador de profundidade da coleção, para determinar os níveis de profundidade ou intensidade dos recursos da coleção. (BIBLARZ, et al. 2001; JOHNSON, 2004; TALAVERA IBARRA, 2005).

O Conspectus é utilizado como um instrumento para análise e avaliação da coleção é uma ferramenta flexível, adaptável e parametrizada e de fácil utilização. É um instrumento para análise da coleção que permite comparar os níveis de profundidade alcançados com as projeções ou metas futuras revisando-as. É utilizado para descrever e caracterizar a coleção, para ajudar na tomada de decisão em relação ao desenvolvimento da coleção. (BIBLARZ, et al. 2001; TALAVERA IBARRA, 2005). 
Além dessa finalidade, o modelo Conspectus atende também à análise da coleção, quer seja durante o processo de elaboração da política, como também para avaliação da coleção, conforme afirma Johnson (2004) que o incluiu entre os métodos de avaliação qualitativa baseados na coleção, processo denominado de mapeamento da coleção.

O Conspectus está automatizado, o que possibilita a coleta de dados específicos das coleções, preparação de estatísticas, alocação de recursos financeiros para diferentes áreas e tomar decisões sobre preservação e armazenamento, pois foram incluídos códigos de preservação. O programa Automated Collection Assessment and Analysis Service (ACAS) utiliza os registros MARC para dar informação sobre a coleção de uma ou de diversas bibliotecas, compará-las, emitir informes em texto ou gráficos e arquivar essas informações (BUSHING, 2001; TALAVERA IBARRA, 2005).

Está disponível no OCLC, o WorldCat Collection Analysis Service, que inclui o OCLC Conspectus. Por meio desse serviço de análise da coleção, mediante um contrato mantido com OCLC, qualquer biblioteca pode, como instituição individual ou como membro de um grupo de bibliotecas, ter acesso ao sistema e analisar o desenvolvimento de sua coleção e comparar com o de outras que integram o consórcio.

Cabe ressaltar que na literatura o Conspectus é tratado como modelo, método, metodologia, ferramenta, percebe-se uma falta de consenso quanto a este aspecto.

\section{CONSPECTUS: INDICADORES DE PROFUNDIDADE}

O Conspectus compreende um conjunto de divisões, categorias e descritores de assunto organizados hierarquicamente. São 24 divisões (grandes áreas temáticas), que foram transformadas em 32 pela OCLC (ver Anexo A), que são subdivididas em categorias (sub-áreas temáticas) em torno de 500 (ver exemplo no Anexo B), que por sua vez também são subdivididas em descritores, hoje aproximadamente 7000.

Já foi realizado pela RLG e WLN a compatibilização entre as divisões, categorias e assuntos com os esquemas de classificação da Library of Congress, da Classificação Decimal de Dewey (CDD) e da National Library of Medicine (NLM), 
que estão disponíveis na Internet (Download the OCLC Conspectus http://www.oclc.org/collectionanalysis/support/default.htm).

Exemplo da Divisão - Ciências biológicas

1st Category $=$ Life Sciences, Biology

2nd Category $=$ Physiology \& Related Subjects

Subject $=$ Animals

Subject $=$ Plants \& Microorganisms

Subject $=$ Anatomy \& Morphology

Subject $=$ Biophysics

Subject $=$ Tissue Biology \& Regional Physiology

Subject $=$ Cell Biology

3rd Category $=$ Biochemistry

Subject $=$ General Topics of Biochemistry

\subsection{Níveis de indicadores de profundidade}

Para cada divisão, categoria ou assunto são utilizados indicadores de profundidade (ver anexo B). O RLG Conspectus incluiu cinco níveis de indicadores de profundidade para descrição da coleção, que são considerados os indicadores básicos (zero, um, dois, três, quatro e cinco). Entretanto, com o objetivo de possibilitar a utilização por outros tipos e tamanhos de bibliotecas o WLN Conspectus incluiu subdivisões $(1 \mathrm{a}, 1 \mathrm{~b}, 2 \mathrm{a}, 2 \mathrm{~b}, 3 \mathrm{a}, 3 \mathrm{~b})$ perfazendo um total de 10 indicadores de profundidade de coleção. As bibliotecas que desejarem utilizar o modelo Conspectus num projeto cooperativo ou individualmente devem determinar, inicialmente, se utilizarão a escala de cinco níveis ou a escala expandida de 10 níveis.

Os indicadores de profundidade da coleção representam um continuum que vai do nível mínimo de informação até o nível abrangente. Esses níveis incrementais não são iguais, no entanto, a partir do momento em que a diferença de um nível para o nível seguinte pode ser mensurado em termos quantitativos e qualitativos, pode ser definida a quantidade de materiais necessários para mover de um nível para o seguinte, ao longo de uma escala ascendente. Cada nível, na sua maioria, inclui todos os elementos, formatos e características dos níveis anteriores, assim é um processo cumulativo. Isso significa que o nível de Pesquisa contém, não somente os elementos 
de definição do nível de Pesquisa (4), mas também todos os elementos de cada nível, ou seja, Informação mínima (1), Informação básica (2) e Estudo (3).

Os indicadores de profundidade são:

Nível 0 (zero) - fora de abrangência (Out of scope)

A biblioteca, intencionalmente, não coleta materiais em nenhum formato sobre um determinado assunto geral ou específico. É importante identificar os temas que a biblioteca não possui e não irá possuir nenhum material, ou seja, que não fazem parte da sua cobertura temática para informar aos seus usuários, bem como para facilitar o intercâmbio e empréstimo entre bibliotecas, dessa forma facilita identificar os temas que são cobertos ou não por uma determinada biblioteca.

Nível 1 (um) - nível mínimo de informação (Minimal level)

São coleções muito limitadas de materiais gerais, podendo incluir monografias e obras de referência, que dão suporte ao atendimento mínimo de solicitações por parte dos usuários, ou seja, no nível mais básico e elementar.

Caso na coleção existam materiais em uma área temática específica a biblioteca possuirá somente uma coleção mínima, em que serão coletadas apenas as fontes gerais, incluindo monografias e obras de referência dessa área temática específica. Não serão coletados os títulos de periódicos especializados relacionados diretamente com o tema.

A coleção deve ser sistemática e freqüentemente revisada para ter somente informação corrente e atualizada. Novas edições devem substituir as antigas, que devem ser retiradas da coleção, também materiais cujo conteúdo esteja desatualizado devem ser excluídos. No entanto, os materiais clássicos ou retrospectivos podem ser mantidos na coleção.

- Nível 1a - nível mínimo de informação, cobertura desigual (uneven coverage)

Poucas seleções e uma representação pouco metódica e não sistemática dos assuntos; os suportes são limitados e há necessidade de serviços específicos; a coleção é coerentemente mantida apesar da cobertura ser limitada.

- Nível 1b - nível mínimo de informação, cobertura focada (focused coverage)

Poucas seleções, mas uma representação sistemática dos assuntos, deve incluir autores básicos, alguns trabalhos clássicos e básicos e um amplo espectro de pontos de vista. A coleção deve ser mantida com coerência. 
Nível 2 (dois) - nível básico de informação (basic information level)

São as coleções que servem para apresentar, introduzir e definir uma área temática, para indicar os distintos tipos de informação disponíveis em outros lugares, para cobrir as necessidades gerais dos usuários da biblioteca, para atender às necessidades de informação de um público que já tenha algum nível educacional e para atender aos alunos que estão cursando os dois primeiros anos da graduação do ensino universitário.

O acervo inclui uma coleção limitada de monografias gerais e obras de referência, uma coleção limitada de periódicos gerais representativos. O acesso a recursos eletrônicos de propriedade da biblioteca ou acessados remotamente, tais como textos, bancos de dados, periódicos são limitados. A coleção deve ser freqüente e sistematicamente revisada para verificar a atualização da coleção. As novas edições devem substituir as demais que devem ser retiradas da coleção. As coleções retiradas podem permanecer no acervo de materiais clássicos, básicos e materiais retrospectivos.

- Nível 2a - nível básico de informação, introdutório (basic information level, introductory)

As coleções limitadas a um nível introdutório da área temática respectiva, composta por monografias e obras de referência, que incluem: trabalhos básicos explicativos, história quanto ao desenvolvimento do tema, trabalhos gerais sobre a área e os personagens mais importantes, enciclopédias gerais, obras de referência, especificamente índices de periódicos e fontes estatísticas. Este nível da coleção é suficiente para apoiar e atender a demandas de usuários em geral e alunos do ensino médio, no sentido de localizar informações gerais sobre um determinado assunto.

- Nível 2b - nível básico de informação, avançado (basic information level, advanced)

Neste nível a coleção inclui periódicos gerais e um amplo e mais profundo conjunto de monografias introdutórias e obras de referência que incluem: trabalhos básicos explanatórios, história do desenvolvimento da área, trabalhos gerais sobre a área e as personalidades importantes na área, um conjunto mais amplo de enciclopédias gerais, índices de periódicos e fontes estatísticas. Inclui uma coleção limitada de periódicos gerais representativos. Define acesso a uma coleção limitada 
de recursos eletrônicos - tanto proprietários como de acesso remoto - de textos, bancos de dados, periódicos etc. Uma coleção nesse nível é suficiente para apoiar a necessidade de informação básica, de leitura e de recreação de um público geral educado ou de estudantes nos dois primeiros anos da universidade.

Nível 3 (três) - nível de apoio instrucional ou estudo (study or instructional support level)

São coleções que fornecem informação sobre um assunto de forma sistemática, mas em um nível mais inferior de intensidade do que para atender à necessidade de informação para pesquisa, dá suporte aos estudantes dos primeiros anos do ensino universitário e demais usuários gerais da biblioteca. $\mathrm{O}$ acervo deve incluir uma coleção extensa de monografias gerais e obras de referência, assim como monografias e obras de referência especializadas selecionadas. Deve incluir uma coleção extensa de periódicos gerais e uma coleção representativa de periódicos especializados. As coleções limitadas de materiais em outros idiomas além do idioma predominante da coleção e do país, por exemplo, materiais para auxiliar na aprendizagem de um idioma, literatura no idioma original, tais como poesia alemã em alemão ou história da Espanha em espanhol. Extensa coleção de trabalhos de autores bem-conhecidos e seleções dos trabalhos de autores menos conhecidos. Deve contemplar a definição de acesso para uma ampla coleção de recursos eletrônicos próprios ou de acesso remoto, incluindo recursos bibliográficos, textos, bancos de dados, periódicos etc. A coleção deve ser revisada sistematicamente para assegurar que somente as informações importantes e essenciais sejam mantidas no acervo, deve incluir um significativo número de materiais retrospectivos.

- Nível 3a - estudo básico ou nível de apoio instrucional (basic study or instructional support level)

Este nível inclui recursos adequados para comunicar e manter conhecimento sobre os temas primários de uma área de assunto. Incluem uma alta porcentagem da literatura mais importante ou trabalhos básicos na área, uma extensa coleção de monografias gerais e obras de referência, de periódicos gerais e índices e resumos. Com relação ao idioma, pode incluir outros idiomas que não o predominante do acervo ou do país, porém o material estará limitado a materiais de aprendizagem de idiomas, deve incluir um conjunto representativo de autores bem conhecidos no 
idioma original, primeiramente para aprendizagem do idioma. Acesso definido para recursos eletrônicos. Esta coleção dá suporte aos cursos de graduação, bem como às necessidades de estudo de alunos no processo de educação continuada.

- Nível 3b - estudo intermediário ou nível de apoio instrucional (intermediate study or instructional support level)

Neste nível a coleção deve manter os recursos adequados para comunicar e atender as demandas em áreas de conhecimento mais especializadas. Deve fornecer cobertura mais abrangente do assunto, com maior amplitude e profundidade. Deve incluir uma alta porcentagem da literatura mais importante ou dos trabalhos básicos da área, incluindo materiais retrospectivos. Deve incluir uma coleção extensa de monografias gerais e obras de referência e um conjunto selecionado de monografias e obras de referência especializadas. Deve incluir uma coleção extensa de periódicos gerais e uma coleção representativa de periódicos especializados, índices e resumos. Deve incluir uma seleção de materiais em outros idiomas, e autores bem conhecidos no idioma do país. Deve definir um amplo acesso para os recursos eletrônicos. Esta coleção fornece apoio aos semestres mais avançados dos cursos de graduação.

- Nível 3c - estudo avançado ou nível de apoio instrucional (advanced study or instructional support level)

Uma coleção nesse nível deve dispor dos recursos adequados para comunicar e manter conhecimento sobre todos os aspectos relativos à área temática, de forma mais intensa que o nível intermediário, porém, menos que o necessário para pesquisa em nível de doutorado ou pesquisa independente. Deve dispor de uma coleção quase completa de trabalhos básicos incluindo quantidades significativas de materiais e recursos retrospectivos. Deve incluir uma ampla coleção de trabalhos especializados de autores menos conhecidos, bem como dos muito conhecidos. Deve ter uma extensa coleção de monografias e obras de referência, tanto gerais como especializadas. Deve ter uma extensa coleção de periódicos gerais e especializados e índices e resumos. Deve ter um conjunto de recursos selecionados em outros idiomas, incluindo autores muito conhecidos no idioma original e uma seleção de materiais por assuntos específicos em outros idiomas. $\mathrm{O}$ acesso aos recursos eletrônicos deve ter o alcance bem amplo para recursos especializados. Esse nível da coleção deve apoiar o 
desenvolvimento de programas em nível de mestrado, bem como outras demandas em nível de especialização.

Nível 4(quatro) - nível de pesquisa (research level)

As coleções nesse nível devem conter as principais fontes necessárias para o desenvolvimento de programas de doutorado e pesquisa independente. Deve incluir uma coleção muito extensa de monografias e obras de referência gerais e especializadas, uma coleção extensa de periódicos gerais e especializados, coleções extensas de materiais apropriados em outros idiomas além da língua predominante do país ou da coleção, e uma coleção extensa de trabalhos de autores muito e pouco conhecidos. Os acessos aos recursos eletrônicos devem ser definidos para uma extensa coleção, desde os recursos de propriedade da unidade de informação quanto os de acesso remoto, incluindo ferramentas bibliográficas, textos, bancos de dados, periódicos etc. O material antigo deve ser mantido no acervo, preservado e conservado, sistematicamente, para atender às necessidades de pesquisa histórica.

Nível 5 (cinco) - nível abrangente (comprehensive)

São as coleções num campo de conhecimento especificamente definido, empenhadas em serem exaustivas até o razoavelmente possível (i.e., coleções especiais), em todos os idiomas aplicáveis. Inclui coleções exaustivas de materiais publicados, e muito exaustivas de manuscritos e em todos os outros formatos pertinentes. Todo o material antigo é retido e é sistematicamente preservado e conservado para servir às necessidades de pesquisa histórica. Uma coleção exaustiva pode servir como uma fonte nacional ou internacional.

\subsection{Indicadores de abrangência da coleção em relação ao idioma}

Os indicadores de idiomas foram revisados com o objetivo de poderem ser utilizados em diversos países e culturas, e se constituem na mais importante mudança introduzida no RLG Conspectus. A abrangência do idioma está ligada, diretamente, com os níveis de indicador de coleção. A extensão da coleção no idioma primário do país e ou da biblioteca, bem como a extensão de outros idiomas ajuda a determinar o nível do indicador de coleção para cada segmento. A cobertura de idioma qualifica e reforça os níveis da coleção. Além do idioma primário ou predominante, outra cobertura de idioma é essencial para coleções nos níveis 3, 4, ou 5. Geralmente, 
presume-se que o mais alto nível de avaliação, é o mais amplo ou de cobertura adicional mais extensa. Os indicadores de idiomas podem ser adicionados aos indicadores de profundidade de coleção para cada nível, tanto para a coleção existente como para assumir um compromisso de aquisição ou atender a metas de desenvolvimento da coleção quando apropriado.

De acordo com BIBLARZ, et al. (2001) há dois tipos de indicadores de idiomas: um desenvolvido pelo RLG e outro pelo WLN, ambos possuem bastante similitude, conforme descrito no quadro 1.

QUADRO 1 Indicadores de Idioma

\begin{tabular}{|c|c|c|c|}
\hline \multicolumn{2}{|c|}{ Código RLG } & \multicolumn{2}{|c|}{ Código WLN } \\
\hline $\begin{array}{r}\mathrm{E} \\
(\text { English) }\end{array}$ & $\begin{array}{l}\text { Materiais no idioma } \\
\text { do país predominam, pouco } \\
\text { ou nenhum material em } \\
\text { outros idiomas, }\end{array}$ & $\begin{array}{r}\mathrm{P} \\
\text { (primary) }\end{array}$ & $\begin{array}{l}\text { O idioma primário é o } \\
\text { idioma predominante no país. } \\
\text { Pouco ou nenhum material } \\
\text { em outro idioma. }\end{array}$ \\
\hline $\begin{array}{r}\mathrm{F} \\
\text { (foreign) }\end{array}$ & $\begin{array}{l}\text { Diversos materiais em } \\
\text { outros idiomas além do } \\
\text { idioma predominante no país. }\end{array}$ & $\begin{array}{c}\mathrm{S} \\
\text { (selected) }\end{array}$ & \begin{tabular}{l}
\multicolumn{3}{c}{ Diversos materiais em } \\
outros idiomas serão \\
incluídos além do idioma \\
predominante no país.
\end{tabular} \\
\hline $\begin{array}{l}\mathrm{W} \\
(\text { wide })^{-}\end{array}$ & $\begin{array}{l}\text { Seleção ampla de } \\
\text { materiais em todos os idiomas } \\
\text { possíveis. Não está prevista } \\
\text { exclusão de materiais devido } \\
\text { ao idioma }\end{array}$ & $\begin{array}{l}\mathrm{W} \\
(\text { wide })^{-}\end{array}$ & $\begin{array}{l}\text { Ampla seleção de } \\
\text { materiais em outros idiomas } \\
\text { representativos. }\end{array}$ \\
\hline $\mathrm{Y}$ & $\begin{array}{l}\text { Materiais, } \\
\text { primeiramente, nos idiomas } \\
\text { estrangeiros. O foco deve } \\
\text { estar especialmente voltado } \\
\text { para a coleta de material na } \\
\text { língua vernácula da área } \\
\text { temática. }\end{array}$ & $\mathrm{X}$ & $\begin{array}{l}\text { O material é } \\
\text { principalmente em um idioma } \\
\text { diferente do idioma primário } \\
\text { da biblioteca ou do país }\end{array}$ \\
\hline
\end{tabular}

Fontes: Elaboração própria a partir dos dados do RLG e WLN. 
Os indicadores de idioma constantes do quadro 1 podem atender à maior parte dos diferentes tipos de bibliotecas. Entretanto, há países onde será necessária a adaptação da ferramenta Conspectus, é o caso do Canadá e Nova Zelândia, que possuem dois idiomas nacionais ou regionais, bem como outros países que também possuem dois idiomas predominantes, tanto oficiais como extra-oficialmente. $\mathrm{O}$ código apresentado a seguir é sugerido como código adicional para ser utilizado em tais circunstâncias. A política de gerenciamento da coleção será o documento em que serão explicitadas as circunstâncias especiais para os idiomas, bem como para outras características da coleção.

$\mathrm{D}=($ dual $)$ duplo idioma ou dois idiomas primários predominantes, com pequeno ou nenhum material em outro idioma.

\section{OUTRAS INFORMAÇÕES SOBRE CONSPECTUS}

De acordo com Biblarz, et al. (2001) os indicadores de profundidade ou níveis são valores numéricos utilizados para descrever as coleções e as metas a serem alcançadas pela biblioteca. São usados para caracterizar três aspectos diferentes do gerenciamento da coleção na divisão, categoria e níveis de assunto: nível atual de coleção (current collection level - CL), compromisso de aquisição (acquisition commitment - AC) e meta de coleção (collection goal - GL).

As definições Conspectus foram elaboradas de forma que possam ser aplicadas uniformemente a todos os assuntos. Entretanto, alguns temas exigem informação em formatos não impressos, tanto no nível básico como nos demais, essas informações podem ser visuais, auditivas e outros formatos não impressos, que deverão ser também coletados, como por exemplo, a dança, música e outras artes, porém não são limitados a esses temas. Para determinar o nível do indicador de profundidade de coleção mais apropriado para temas nos quais os recursos não impressos são essenciais, deve-se adicionar uma frase "mídias não impressas apropriadas" à linha que detalha a coleta de monografias e obras de referência. Por exemplo, no Nível Básico de Informação (1) para uma coleção de música deverá ser incluída a frase "uma coleção limitada de monografias, obras de referência e mídias não impressas apropriadas". 
Quanto aos recursos eletrônicos relevantes o processo é equivalente ao dispensado aos materiais impressos em qualquer nível. Entretanto, devem ser observados os limites definidos pelas políticas, procedimentos e licenças de uso. Os periódicos eletrônicos, tanto de acesso remoto como os armazenados no local são tratados de forma equivalente aos periódicos impressos se: o acesso aos recursos eletrônicos for pelo menos igual ao acesso ao produto impresso (incluindo gráficos, mapas e outras características); houver acesso a um número suficiente de terminais e linhas; a informação é fornecida sem nenhum custo adicional para o usuário. Da mesma forma, um texto completo eletrônico (full text) de monografias, periódicos, imagens etc., se carregado localmente ou acessado por meio da Internet, é tratado da mesma forma que o formato impresso desde que não tenha nenhum custo adicional para o usuário.

\section{CONCLUSÃO}

Diversas bibliotecas de diferentes países utilizam o Conspectus como instrumento de desenvolvimento e avaliação de coleções, para facilitar a colaboração entre bibliotecas e redes ou para o gerenciamento de suas coleções.

Pode-se constatar que o Conspectus apresenta definições genéricas, e conforme afirma Biblarz, et al. (2001) é necessário o desenvolvimento de guidelines, manuais e políticas de forma a comunicar os consensos na sua aplicação em áreas específicas, como material de ficção, literatura infantil, arquitetura etc. o que não inviabiliza o seu uso, ao contrário possibilita uma utilização muito mais ampla.

Por outro lado, as possibilidades de aplicação do método Conspectus para auxiliar no desenvolvimento e gerenciamento de coleções de bibliotecas de qualquer tamanho e tipo são consideráveis. Pode-se aplicar os cinco níveis básicos a uma única biblioteca, mapeando as áreas temáticas núcleo, as quais deverão ser tratadas nos níveis três ou quatro, ou até mesmo no nível cinco que significa cobrir de forma exaustiva uma determinada área temática. Pode-se, também, identificar as áreas periféricas às quais a coleção deverá atender de forma superficial, facilitando, desta forma, a identificação de outras instituições especializadas nestas áreas para suprir as necessidades de informação. 
Observa-se que a metodologia é interessante, tem um potencial significativo que pode instrumentalizar o desenvolvimento e gerenciamento de coleções. É necessário discutir, aplicar, testar o uso desta metodologia, como também, analisar relatos de experiências quanto ao seu uso. Este artigo consiste na primeira contribuição para discussão. 


\section{REFERENCIAS}

ANDOVER Harvard Theological Library. Disponível em: $<$ http://www.hds.harvard.edu/library/collections/strengths/collection_development.ht $\underline{\mathrm{ml}}>$. Acesso em: 16 maio 2007.

BIBLARZ, Dora; et al. Guidelines for collection development policy using the Conspectus model. IFLA, 2001. Disponível em: 〈http://www.ifla.org/VII/s14〉. Acesso em: 28 abr. 2007.

BRIEF history of the RLG Conspectus. 1997. Disponível em: <http://www.rlg.org/conspechist.html >.Acesso em: 05 jun. 2007.

BUSHING, Mary C. The evolution of Conspectus practice in libraries: the beginnings and the present applications. 2001. 7p. Disponível em: <http://klement.nkp.cz/Caslin/caslin01/sbornik/conspectus.html >. Acesso em: 08 maio 2007. Collection policy for the Howard Ross Library of Management. Disponível em: <http://www.library.mcgill.ca/manage/mgmt.htm > Acesso em 26 ago. 2007.

COLUMBIA University Libraries. Disponível em: <http://www.columbia.edu/cu/lweb/services/colldev/index.html > . Acesso em: 27 ago. 2007

EVANS, G. Edward; SAPONARO, Margaret Zarnosky. Developing library and information center collections. 5.ed. Westport: Libraries Unlimited, c2005. 446p.

JOHNSON, Peggy. Fundamentals of collection development and management.Chicago: ALA, 2004.342p.

LIBRARY of Congress. Disponível em: <http://www.loc.gov/acq/devpol/cpc.html $>$. Acesso em: 05 maio 2007.

MCGILL LIBRARY. Disponível em: 〈http://www.mcgill.ca/library/; http://www.mcgill.ca/library-using/policies/collection/levels/>. Acesso em: 28 ago. 2007;

MUNROE, Mary H.; STEEG, Jennie E. Ver. The decision-making process in conspectus evaluation os collections: the quest for certainty. The Library Quarterly, v. 74, n.2, p. 181-205, Apr. 2004.

OCLC. Disponível em: <http://www.oclc.org/collectionanalysis/support/default.htm>. Acesso em 05 maio 2007

OCLC. WorldCat collection analysis user guide. 2.ed. 2007 . 154p. Disponível em: $<$ http://www.oclc.org/support/documentation/collectionanalysis/using/>. Acesso em: 15 jul. 2007. 


\section{ARTIGO}

TALAVERA IBARRA, Ana Maria. Desarrollo de colecciones em bibliotecas universitárias: revisando Conspectus. In. Proceedings. Jornadas Nacionales de Bibliotecas Universitárias, 2. Trujillo, Peru, 26-27 ago. 2005. 18p. Disponível em: <http://eprints.rclis.org/archive/00009064/>. Acesso em: 05 maio 2007. 
Anexo A - As 32 divisões temáticas do Conspectus são:

\begin{tabular}{|c|c|c|}
\hline Divisão & $\begin{array}{l}\text { Prefixo } \\
\text { LC }\end{array}$ & $\begin{array}{l}\text { Prefixo } \\
\text { Dewey }\end{array}$ \\
\hline AGRICULTURA & AGR & AGD \\
\hline ANTROPOLOGIA & ANT & AND \\
\hline ARTE E ARQUITETURA & ART & ARD \\
\hline ARTES CÊNICAS & PER & PED \\
\hline $\begin{array}{lcc}\text { BIBLIOTECONOMIA } & \text { (LIBRARY } & \text { SCIENCE), } \\
\text { GENERALIDADES E REFERÊNCIA } & \end{array}$ & LIS & DLS \\
\hline CIÊNCIAS BIOLOGICAS & $\mathrm{BIO}$ & BID \\
\hline CIÊNCIA DA COMPUTAÇÃO & $\mathrm{COM}$ & CSD \\
\hline CIÊNCIAS FISICAS & PHY & PUD \\
\hline \multicolumn{3}{|l|}{ CIÊNCIAS PRÉ-CLINICAS } \\
\hline CIÊNCIAS POLÍTICAS & POL & POD \\
\hline COMÉRCIO E ECONOMIA & ECO & BUD \\
\hline DIREITO (LEGISLAÇÃO) & LAW & DLA \\
\hline \multicolumn{3}{|l|}{ DOCUMENTOS DO GOVERNO } \\
\hline \multicolumn{3}{|l|}{ DOENÇAS TRANSMISSÍVEIS E MISCELÂNEAS } \\
\hline EDUCAÇÃO & EDU & EDD \\
\hline EDUCAÇÃO FÍSICA E RECREAÇÃO & PHR & $\mathrm{DPH}$ \\
\hline ENGENHARIA E TECNOLOGIA & TEC & END \\
\hline FILOSOFIA E RELIGIÃO & PAR & PHD \\
\hline GEOGRAFIA E CIÊNCIAS DA TERRA & GEO & GED \\
\hline HISTÓRIA E CIÊNCIAS AUXILIARES & HIS & HID \\
\hline \multicolumn{3}{|l|}{$\begin{array}{l}\text { INSTALAÇÕES DE SAUDE, ENFERMAGEM E } \\
\text { HISTÓRIA }\end{array}$} \\
\hline LÍNGUA, LINGUÍSTICA E LITERATURA & LLL & LAD \\
\hline MATEMÁTICA & MAT & MAD \\
\hline MEDICINA & MED & DME \\
\hline \multicolumn{3}{|l|}{ MEDICINA POR SISTEMA DO CORPO HUMANO } \\
\hline \multicolumn{3}{|l|}{ MEDICINA POR DISCIPLINA } \\
\hline MUSICA & MUS & MUD \\
\hline PSICOLOGIA & PSY & PSD \\
\hline QUIMICA & $\mathrm{CHE}$ & $\mathrm{CHD}$ \\
\hline \multicolumn{3}{|l|}{ SAÚDE OCUPACIONAL E SAÚDE PÚBLICA } \\
\hline SOCIOLOGIA & $\mathrm{SOC}$ & SOD \\
\hline CLASSIFICAÇÕES DESCONHECIDAS & & \\
\hline
\end{tabular}


Anexo B - Exemplo da utilização do Conspectus pela Andover-Harvard Theological

Library

\begin{tabular}{|c|c|c|c|}
\hline \multicolumn{2}{|c|}{ LC Classification } & $\begin{array}{l}\text { Collecting } \\
\text { Level }\end{array}$ & \multirow{2}{*}{\begin{tabular}{|l} 
Notes \\
Research collections at \\
Widener and other \\
libraries
\end{tabular}} \\
\hline B-BJ & Philosophy and Psychology & $\begin{array}{l}4 \text { Except } \\
\text { for the } \\
\text { following: }\end{array}$ & \\
\hline B56 & $\begin{array}{l}\text { Philosophy in relation to theology } \\
\text { and religion }\end{array}$ & 3 & \\
\hline BJ47 & Ethics in relation to religion & 3 & \\
\hline $\begin{array}{l}\text { BJ1188- } \\
\text { BJ1278 }\end{array}$ & Christian Ethics & 2 & \\
\hline BL & Religions, mythology, rationalism & 3 & $\begin{array}{l}\text { Research collections at } \\
\text { Widener and other } \\
\text { libraries }\end{array}$ \\
\hline BM & Judaism & & $\begin{array}{l}\text { Research collections at } \\
\text { Widener }\end{array}$ \\
\hline $1-159$ & General & 4 & \\
\hline $160-178$ & Ancient period & 2 & \\
\hline $180-449$ & Medieval, modern & 4 & \\
\hline $480-485$ & Pre-Talmud, non-biblical literature & 4 & \\
\hline $487-488$ & Dead Sea Scrolls & 2 & \\
\hline $495-534$ & Midrash, Cabala, etc. & 4 & \\
\hline 535 & Jews and Christianity & 2 & \\
\hline $536-585$ & $\begin{array}{l}\text { Relations of Judaism to special } \\
\text { subject fields }\end{array}$ & 4 & \\
\hline 590 & Jewish works against Christianity & 2 & \\
\hline $590-601$ & Dogmatic Judaism & 4 & \\
\hline $605-630$ & $\begin{array}{l}\text { Theology of the OT in Jewish } \\
\text { teaching }\end{array}$ & 3 & \\
\hline $645-755$ & Dogmatic Judaism, Practical & 4 & \\
\hline $900-990$ & Samaritans & 2 & \\
\hline BP & Islam, Bahaism, Theosophy, etc. & 4 & $\begin{array}{l}\text { Research collections at } \\
\text { Widener }\end{array}$ \\
\hline BQ & Buddhism & 4 & $\begin{array}{l}\text { Research collections at } \\
\text { Widener and Harvard- } \\
\text { Yenching. }\end{array}$ \\
\hline $\mathbf{B R}$ & Christianity & & \\
\hline $1-67$ & General: Patristics & 2 & \\
\hline $75-100$ & Later writers & $2-3$ & $\begin{array}{ll}\text { Catholics } & (3) ; \\
\text { Protestants (2) }\end{array}$ \\
\hline
\end{tabular}




\begin{tabular}{|l|l|l|l|}
\hline $110-114$ & Philosophy of Christianity & 3 & \\
\hline $115-225$ & $\begin{array}{l}\text { Christianity in relation to special } \\
\text { subjects: History of Christianity to } \\
451\end{array}$ & 2 & \\
\hline $227-275$ & Christianity in the Middle Ages & 4 & \\
\hline $280-355$ & Reformation & 2 & \\
\hline $\begin{array}{l}358- \\
1609\end{array}$ & Christianity by country & $3-2$ & $\begin{array}{l}\text { Catholic history (3) } \\
\text { Protestant (2) }\end{array}$ \\
\hline $\begin{array}{l}1610- \\
1653\end{array}$ & Liberalism, toleration, pietism & 2 & \\
\hline $\begin{array}{l}1690- \\
1719\end{array}$ & $\begin{array}{l}\text { Religious biography, general \& } \\
\text { juvenile }\end{array}$ & 4 & \\
\hline $\begin{array}{l}1720- \\
1725\end{array}$ & $\begin{array}{l}\text { Early Christian biography to ca. } \\
600\end{array}$ & 2 & \\
\hline
\end{tabular}

Fonte: Andover-Harvard Theological Library - http://www.hds.harvard.edu/library/ collections/strengths/collection_development.html

Como citar este artigo:

CARIBÉ, Rita de Cássia do Valé Caribé. Conspectus: um método para o gerenciamento de coleções em bibliotecas. Rev. digit. bibliotecon. cienc. inf., Campinas, SP, v.12, n.1, p.39-60, jan/abr. 2014. ISSN 1678-765X. Disponível em:

<http://www.sbu.unicamp.br/seer/ojs/index.php/rbci>. Acesso em: 30 jan. 2014. 